\title{
A Real-Time Open Access Platform towards Proof of Concept for Smart Grid Applications
}

\author{
Mohammed Kemal ${ }^{1}$, Lennart Petersen ${ }^{2}$, Florin Iov ${ }^{2}$, \\ and Rasmus Løvenstein Olsen ${ }^{1}$ \\ ${ }^{1}$ Department of Electronic Systems, Aalborg University, Denmark \\ 2 Department of Energy and Technology, Aalborg University, Denmark \\ E-mail:seifu@es.aau.dk; rlo@es.aau.dk; lep@et.aau.dk; fi@et.aau.dk
}

Received 28 December 2016; Accepted 30 May 2017;

Publication 19 February 2018

\begin{abstract}
This paper presents development of real time open access platform towards proof of concept of smart grid applications deployed at Smart Energy System Laboratory of Aalborg University. Discussed on the paper is the architecture and set-up of the platform by elaborating the three main layers: electrical grid layer, ICT \& network emulation layer and control layer. DiSC-OPAL, a toolbox built for OPAL-RT real time grid simulation; comprising of models for wide variety of controllable flexible assets, stochastic power sources for wind and solar power plants, real consumption data's and electrical grid components is presented. A detailed model description of the whole set up and the corresponding functionalities is characterized. To showcase real life application of the whole framework, an overview of two test cases implemented for European SmartC2Net project with focus on control and market integration of low voltage distribution grids is presented.
\end{abstract}

Keywords: Network Emulation, Real Time Simulations, DLMS, OADR, Virtual Smart Meters, Demand Response, Voltage Control, Smart Grid, Power Communication Co-Simulation.

Journal of Mobile Multimedia, Vol. 14_1, 49-74.

doi: 10.13052/jmm1550-4646.1413

This is an Open Access publication. (c) 2018 the Author(s). All rights reserved. 


\section{Introduction}

The current fast evolving global intelligent energy system is facing a daunting task of accommodating the major players on energy system infrastructures, involving, electricity from clean energy sources, intelligent supply of thermal energy, advanced energy storage facilities and smart integration of the transportation sectors. It has become apparent to transform the energy system planning and strategies for environmentally friendly and sustainable future $[1,2]$.

In 2015, Danish wind power generation units produced what corresponds to record breaking $42 \%$ of the country's electricity consumption. In Jutland and Funen regions wind power, in fact, produced more electricity than the total consumption of 1,460 hours of the year [4]. After the historical 2012 Danish energy agreement with an ambitious goal of supplying $50 \%$ of the energy consumption from renewable energy sources, the penetration of distributed photo-voltaic (PV) systems and small wind turbines is increasing at a rapid rate.

This foreseen highly increasing penetration of wind and PV into the Danish electricity supply imposes the requirement that this large scale renewable generation shall contribute to grid support services such as voltage, frequency and rotor angle stability control in order to ensure the system stability comprising both transmission and distribution level. In particular, the dispersed pattern of wind turbines and PV systems in distribution networks requires coordination of various controls with fast and reliable communication (i.e. between wind power plants and the system operator) to exploit those flexible assets in the medium-voltage (MV) grids [5, 6].

The high complexity of the aforementioned factors calls for advanced design and testing methods. Real-time (RT) simulators coupled with ICT and communication network emulation platform can be crucial in this regard, enabling researchers to test complex systems and methods related to smart grid development directly in their laboratories [3]. There is an urgent need for platforms that can take in to account both ICT layer and real-time grid simulation platform with capabilities to incorporate the key players across all related sectors and create an optimal environment to test new research methods, technologies and practices.

Smart Energy System Laboratory of Aalborg University (Figure 2) is built with a major goal to create a platform that can integrate the existing smart energy system demonstrators, real-time hardware in the loop (HIL) platforms, 


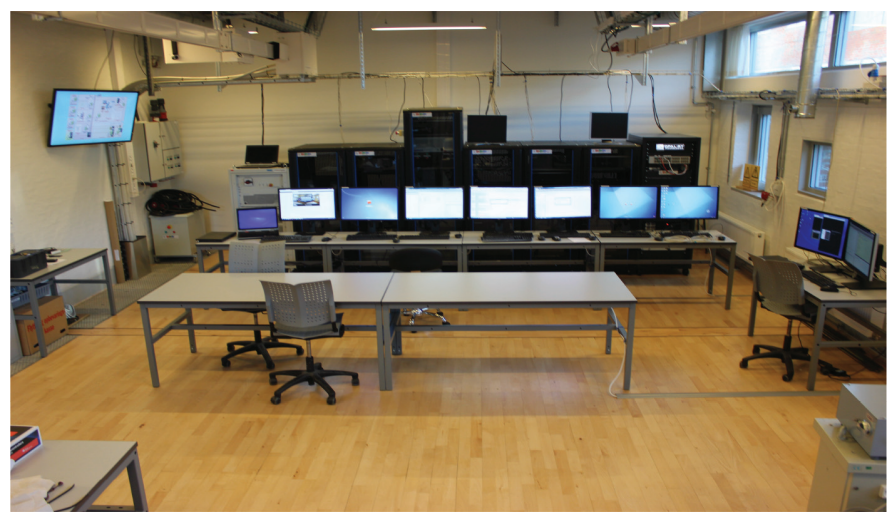

Figure 1 Smart Energy System Laboratory Setup [2].

and involving all related stakeholders. This framework is unique because it can be used to incorporate aspects of power system, ICT \& communication network and control functionalities simultaneously in real-time. Experimental platforms of this type would provide functionalities to perform complex experiments which would otherwise be impossible to test on a real life conditions.

The paper is structured as follows; Section 2 illustrates the whole architecture and detailed overview of the layers in the Smart Energy Systems Laboratory platform. In Section 3, practical applications of the setup dealing with Active Power Management, Demand Response platform and Wind Power Plant Voltage Control is presented. Finally Section 4, summarizes the paper.

\section{Smart Energy Systems Laborartory Architechture}

Smart Energy Systems Laboratory setup shown at Figure 2 is established with the vision to capture an integrated simulation of power and communication networks by using real life actual data to build and test new research findings and technologies.

It is constructed to support the three key layers in intelligent power systems: the electrical grid, ICT with communication network emulation, and the control layer. DiSC-OPAL library, development of a dynamic SIMULINK library toolbox built for OPAL-RT real-time simulation environment is also presented here. 
52 M. Kemal et al.

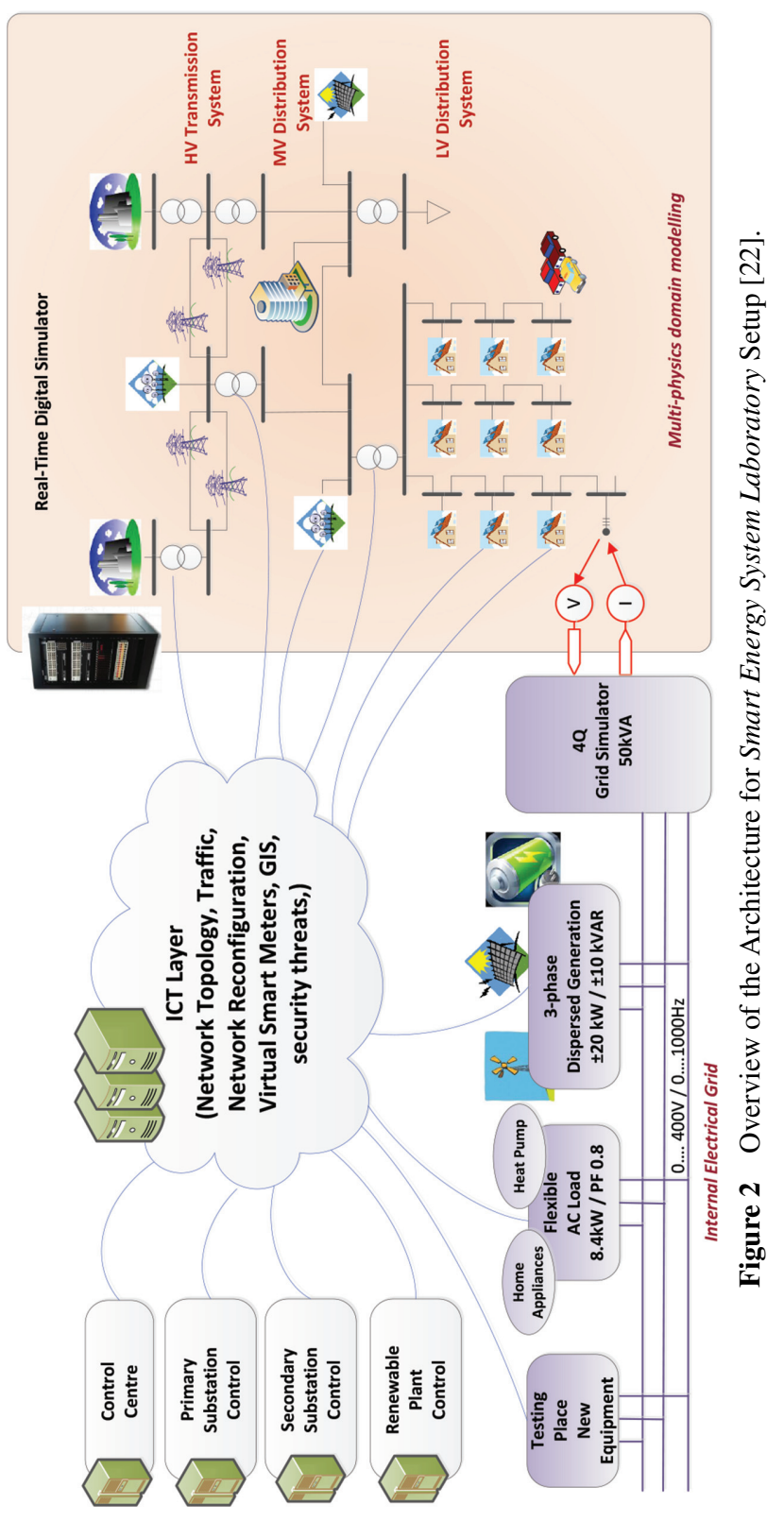




\subsection{Electrical GRID}

The main electrical grid components as seen in Figure 2 at Smart Energy Systems Laboratory setup are presented as follows,

\subsubsection{OPAL-RT real time simulator}

Inputs from a real-time digital simulator based on OPAL-RT technology is realizing the simulation of large scale energy networks including various systems using the multi-domain physics approach. Both electrical networks as well as thermal and mechanical systems can be incorporated. However, the main goal for this real-time simulator in Smart Energy System Laboratory is to capture the electrical system from the transmission level (TSO) down to low voltage distribution grids (DSO). The power system algorithms produce output conditions that realistically represent conditions in a real network, making real-time simulation significant for two reasons; the user is capable of performing HIL testing such as with industrial controllers (see Section D) and realistic communication networks can be incorporated as interface between simulated electrical network and the physical components (see Section C). The OPAL-RT is able to simulate up to 10000 three-phase buses in RMS and 600 nodes in EMT. Implementation of all models is based on Matlab/ Simulink.

For the test-cases in Section 3, a medium voltage and low voltage network as part of a benchmark distribution grid used for SmartC2net is implemented on OPAL-RT simulation platform. Controllable and uncontrollable assets are added to the benchmark grid, as shown in Figure 3 (MV grid) and Figure 4 (LV grid). SPP is a $3 \mathrm{MW}$ solar power plant, WPP is a $2.7 \mathrm{MW}$ wind power plant. The low voltage grid controller (LVGC) is managing a LV grid at Bus B10; There are three controllable assets in the MV grid, PV plant connected to bus B4, a WPP connected to bus B11, and a low voltage grid (controlled through the LVGC at bus B10). All the remaining consumptions and productions in the MV grid are implemented according to realistic load profiles.

The controllable assets in the LV grid are three PV systems combined with energy storages. The uncontrollable consumption and production comprise household consumption and five uncontrollable PV systems. The data for the household consumption is given by traces from real consumption data provided in DiSC-OPAL toolbox (see Section 2.2), which also provides the asset models used for the implementation. 


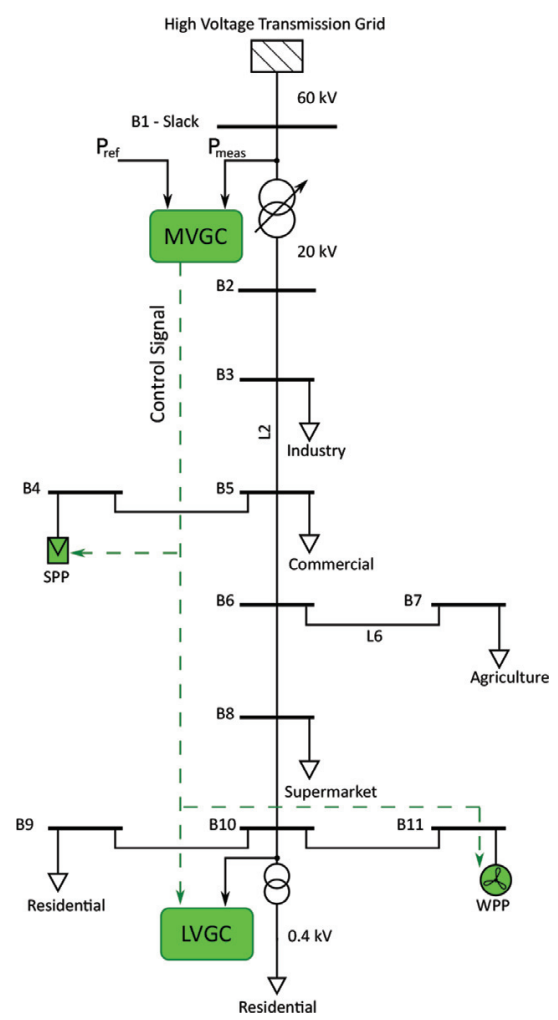

Figure 3 Medium voltage distribution grid.

\subsubsection{Internal electrical grid}

The 3-phase voltages measured in a given point in the simulated distribution network in OPAL-RT can be applied to the $50 \mathrm{kVAAC} / \mathrm{DC}$ Four Quadrant grid simulator supplying the physical components i.e. dispersed energy resource, flexible load as they are part of the larger system (see Figure 2). The three phase currents are fed in back to the real-time digital simulator. A fully regenerative four quadrant power converter is emulating the dispersed generation unit. It has $20 \mathrm{~kW} / 10 \mathrm{kVAR}$ capability and it is used to mimic characteristics of a small wind turbine, a PV systems or energy storage. Several controllable flexible AC/DC Loads are used to mimic the behavior of loads in a typical household. This system is receiving set-points from hierarchical control structure (see Section D). Different smart meter technologies are installed on the platform and can provide power and energy consumption from the physical assets to the upper hierarchical control levels. 


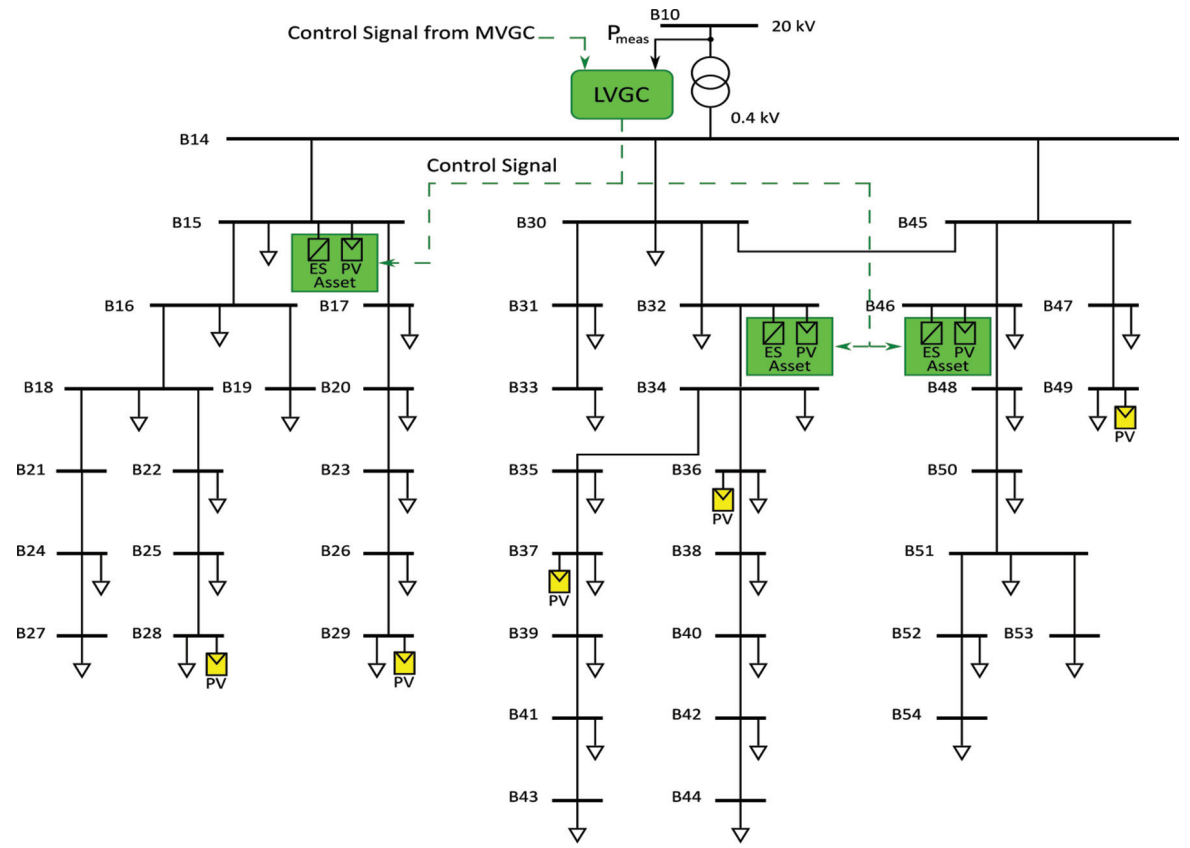

Figure 4 Low voltage distribution grid.

\subsection{DiSC-OPAL Toolbox}

DiSC-OPAL Toolbox is primarily used to verify voltage control algorithms in power distribution grids during real-time simulations using OPAL-RT, for bandwidth range of less than $0.2 \mathrm{~Hz}$. The toolbox comprises SIMULINK models for wide variety of controllable flexible assets, stochastic power sources for wind and PV power plants, real consumption data and electrical grid components (see Figure 3). This toolbox is an extension of [5] and [10], a MATLAB based simulation framework originally built to verify voltage control approaches on power distribution systems. DiSC is primarily developed due to the unavailability of similar simulation platforms that can support flexible assets with grid control, models with dynamic consumption patterns and specifically targeted to study voltage control problems. The full platform and related documentation is available on the homepage [10].

\subsection{ICT and Network Emulation}

Intelligent energy systems benefit from modern ICT and communication network advancements to ensure the exchange of information used to update and 


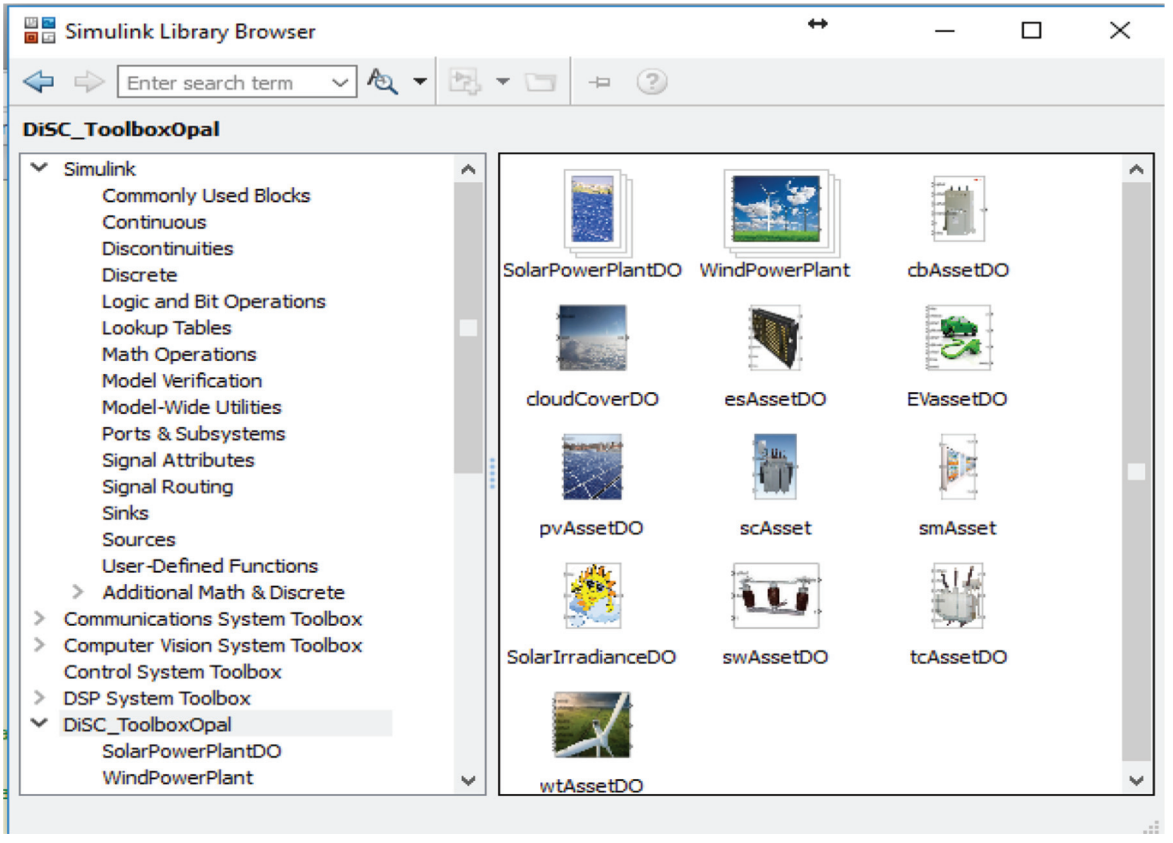

Figure 5 A snapshot of DiSC Toolbox for OPAL-RT based on MATLAB/SIMULINK environment [11].

monitor the status of each component in the power generation, transmission and distribution systems. This information is later used in making decisions used for management, monitoring and control functionalities.

The physical layout of the ICT layer in the Smart Energy System Laboratory is shown on Figure 6. All computers and hardware components are connected via Gigabit Ethernet local area network (LAN) dedicated only for the laboratory giving the setup a high level of flexibility and room for controlled expansion. Adetailed description of the core components is outlined as follows.

\subsubsection{Cisco catalyst 2960xseries switch}

In the middle core of the set up is Cisco Catalyst 2960xseries stackable layer 2 and layer 3 access switch. This device is chosen due to its ease of deployment, management and troubleshooting. It is highly secure in that it uses standards based 802.1x for port based network access control, capable of scalable and dynamic role based access control with Cisco TrustSec and protects against IPV6 address theft attacks with IPV6 frist hope security [20]. The Switch is 


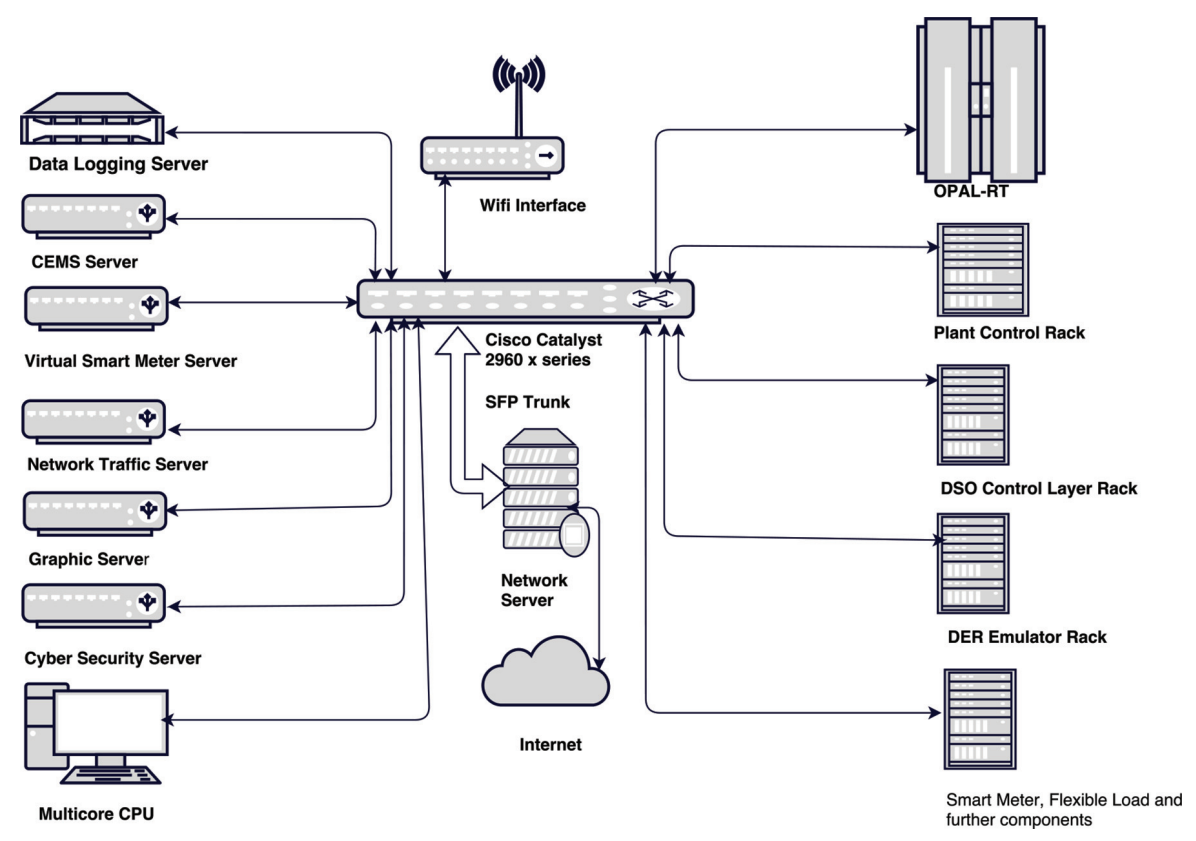

Figure 6 Overview of the ICT Setup for Smart Energy System Laboratory Setup.

configured with a separate VLAN on ports 2-48. Port 1, gigabyte Ethernet small form-factor pluggable (SFP+1) and $\mathrm{SFP}+2$ are all set up as trunks meaning that all traffic on ports $2-48$ will be routed though one of the trunks to the network server and not directly between ports.

\subsubsection{Network server}

The network server is running Ubuntu Linux operating system which gives flexibility and serve for several purposes in the laboratory. It functions as a router providing Internet and NAT services for the local LAN. Moreover, it provides DHCP functionality, logs network traffic, provides NTP services, and serves as a network emulator for studying different communication technologies. The network emulator is designed to accommodate state-of-the-art network emulation and simulation platforms like KauNet, OMNeT++, OP-NET and NS3. The Network Server provides additional functionality of using $\mathrm{SSH}$ for remote access and configuration of the whole setup.

Currently KauNet communication network emulation platform is configured on the system and elaborated in [18]. It is chosen because of its capability 
to perform network modeling with large degree of control and repeatability. It also gives the highly desired functionality of placing deterministic delay and packet loss patterns as well as precise control over bandwidth and delay changes [18] and [19].

\subsubsection{CEMS server}

Customer Energy Management System (CEMS) server is dedicated for an application service that communicates with household devices for demand management systems. The platform was primarily implemented for SmartC2Net project as part of demand management control system to address situations where household consumption loads shift in timely manner [22]. This server is built with capability to configure one or many virtual CEMS machines and talk with the high layer demand response platform using the standard OpenADR protocol. It gives the system high degree of flexibility in that it mimics a platform with multiple virtual CEMS models in one machine and which appear as multiple independent CEMS devices to the upper layer demand management controller. CEMS runs optimization algorithm for customer energy managment by using generic Gurobi optimization tool [21].

\subsubsection{Virtual smart meter server}

Similar to CEMS server, virtual smart meter server is also designed and implemented as an integral part of European SmartC2net project platform intended for demand management control functionality. It is developed to mimic multiple real smart meters and by using the standard Device Language Message Specification (DLMS) protocol to exchange information with $G$-Smart, an industrial data aggregation hardware developed by EFACEC [22]. For the aforementioned use case, G-Smart was used for demand management control HIL platform implementation. The virtual smart meter platform enables simulation based evaluation of different control and automation strategies to the demand response system.

\subsubsection{Visualization server}

The graphics visualization server is used to facilitate automation of different smart grid applications implemented in the set up. It is previously used for online configuration of the network emulation server, where further information can be found in [18]. It is built to be flexible in a way that it can be utilized to model SCADA system for DSO voltage regulations, SCADA system for wind power plants and automation functionalities with GIS mapping. 


\subsection{Control Layer}

The control layer has components to model and analyse demand response platform (DR, see Section 3.2), automation and control of primary and secondary substations, medium voltage grid controller, low voltage grid controller and plant control unit. Implementation of models as well as controls is done using MATLAB/SIMULINK. It is modelled by using hierarchical control mechanism which offers the ability of decomposing the complexity into smaller sub-problems.

Hierarchical control structure, as shown in Figure 7, operates on several levels; the central management level, the medium voltage level, the low voltage level and the household level. This model breaks down the overall complexity of the system in to the hierarchies specified in the system.

For the structure of the electrical grid implemented, a control layer is added where some of the control is distributed throughout the distribution grid in contrast to the traditional centralized system. The medium voltage grid controller and low voltage grid controllers handle any voltage issues locally and provide flexibility at the same time to the upper layers Figure 7.

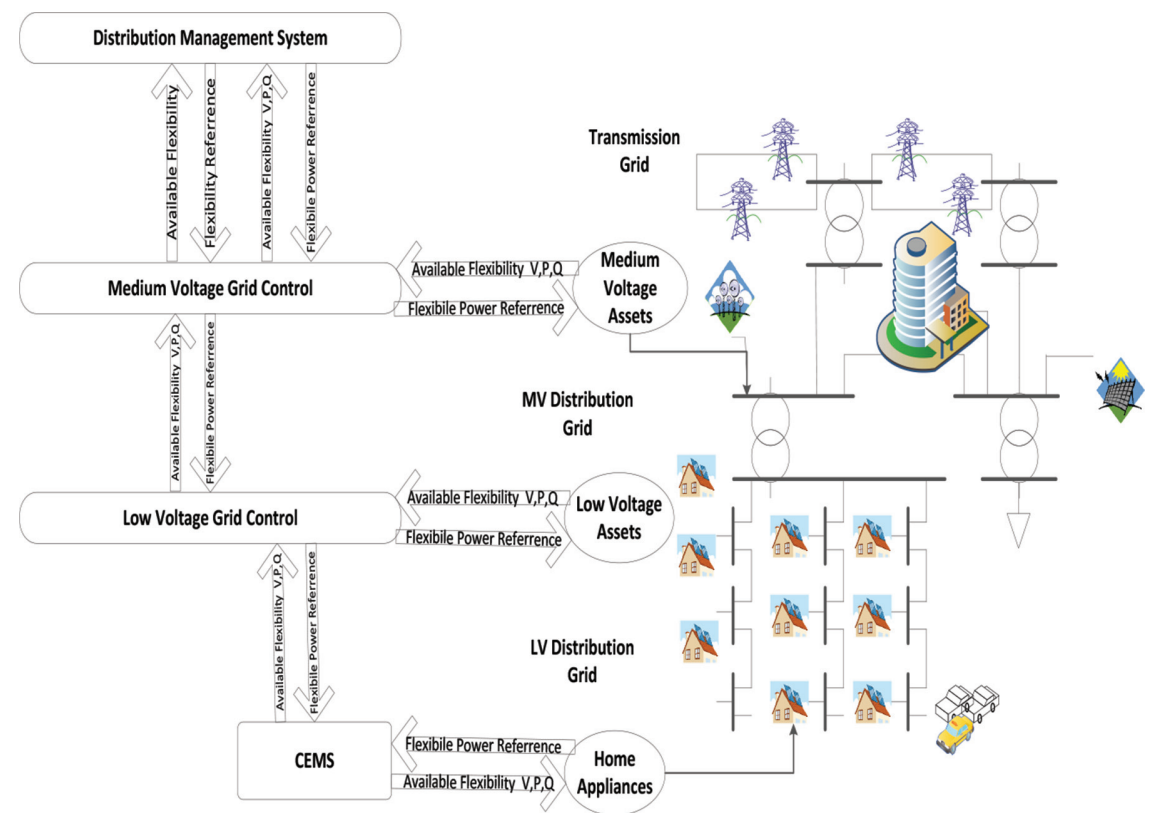

Figure 7 Implemented hierarchical control model. 
The functionalities shown in Figure 7 are executed on physical machines located on multiple locations, which requires communication to be able to receive inputs and send set points between each other. However, as different controllers have varying quality of service (QOS) requirements which is dependent on the communication technology used, ICT platform of Smart Energy System Laboratory is essential in emulating this technologies.

\section{Real Time Test Cases}

To give us a better perspective of how Smart Energy System Laboratory can be employed to study Smart Grid applications involving ICT and real-time hardware in the loop platforms, the following test cases are presented.

\subsection{Active Power Management in Distribution Grid Test Case}

The functionalities validated in this test-case are:

- Impact of Communication Technologies: PLC, GPRS and LTE communication technologies are compared and the impact on the controller is studied. Real experimental traces for delay, packet loss, bandwidth and cross traffic is used to create a realistic real-time network emulation platform.

- Energy balancing: It is shown that a distribution grid can be controlled to follow an active energy reference. The performance of the energy balancing is evaluated according to root mean square error of reference tracking [22].

- Loss Minimization: It is shown that active power losses in the MV grid can be reduced by making use of the medium voltage controller.

\subsubsection{Active power management test result}

To capture the full day disparity on consumption and production, each of the conducted tests has duration of 16 hours. For verification purposes, the MV grid is controlled to follow an active power reference. The controllable assets are used to compensate for the variations and follow the reference accordingly. The following scenarios are considered for the tests:

- Energy balancing with ideal communication (no loss introduced by the network emulator, Ethernet in the local LAN). 
- Energy balancing with imperfect communication (PLC, Gprs, LTE technologies emulated).

- Energy balancing with imperfect communication and controller gain adaptation.

One result of the numerous test cases studied is shown in Figures 8 and 9 compares the reference tracking ability for LVGC and MVGC by using ideal communication (only Ethernet LAN) and non ideal communication (PLC with $7 \mathrm{kbps}$ cross traffic). For the ideal case on LVGC, an average error of $4.5 \%$ is measured, while for the non ideal case the average error rises to $15.1 \%$. From this, it is concluded that the tracking ability of LVGC tested with PLC with $7 \mathrm{kbps}$ is acceptable for power balancing control functionality satisfying requirement of the controller used for the experiment [22].

The MVGC reference following capacity has not been affected as the LVGC case because ideal communication is considered between LVGC and MVGC for this specific scenario. This shows the impact of PLC communication with $7 \mathrm{kbps}$ between LVGC controller and flexible assets has minimal impact on the higher layer MVGC. Further results of the remaining test cases are found at [22] and will be addressed on subsequent publications.
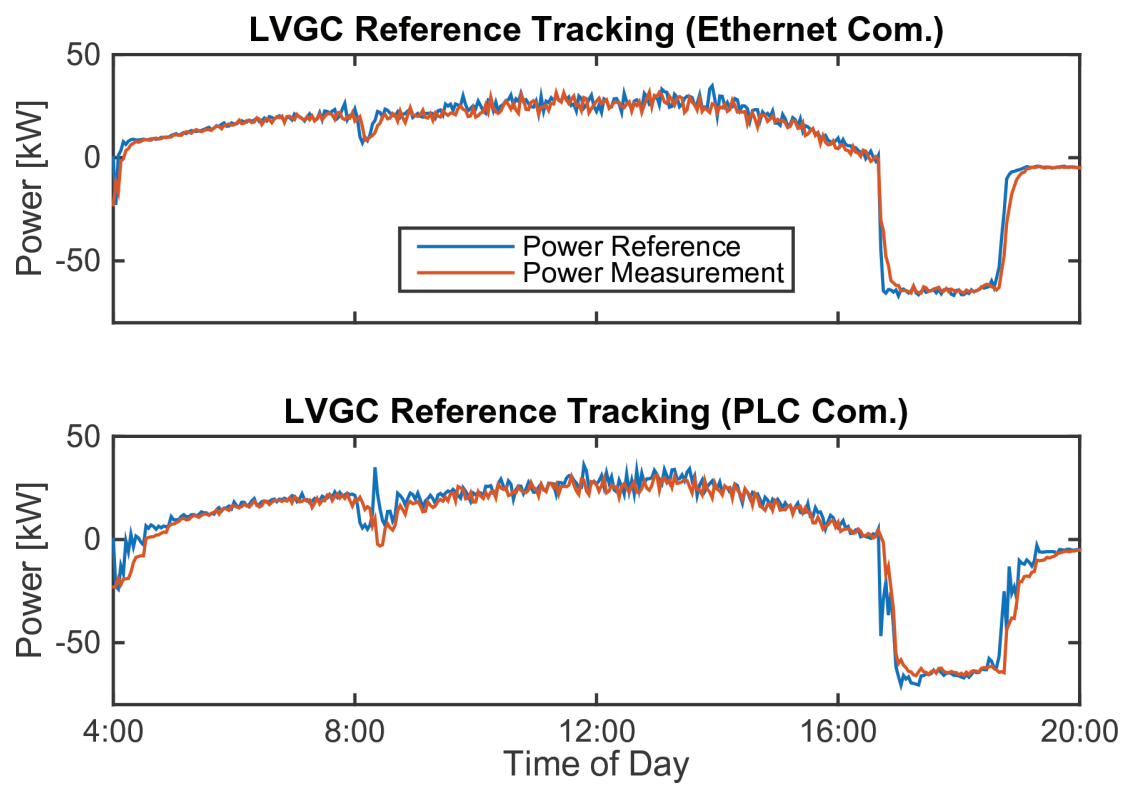

Figure 8 Reference tracking of low voltage grid. 


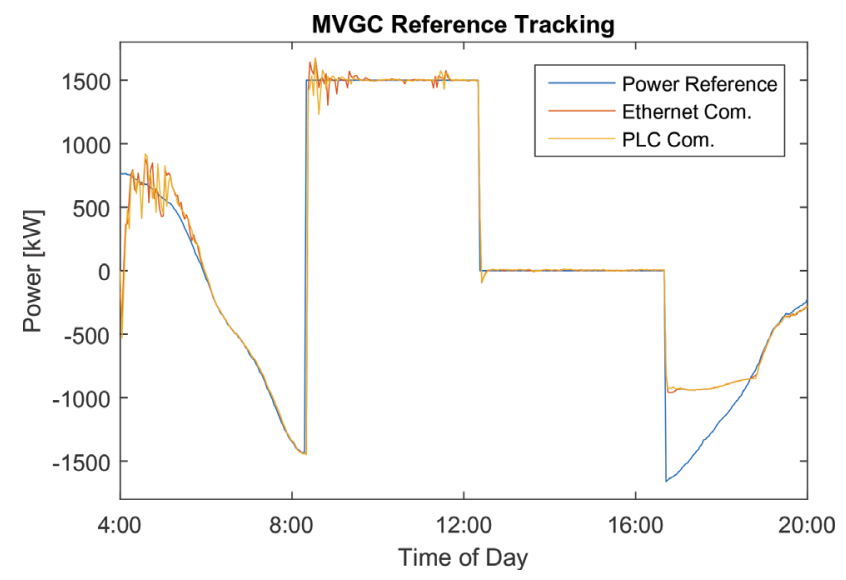

Figure 9 Reference tracking of medium voltage grid.

\subsection{Demand Management Platform}

\subsubsection{Demand management test case}

Demand Management is a planning methodology that addresses the case of shifting electric consumptions in response to electricity prices by making use of proper scheduling mechanisms. Smart Energy System Laboratory is used to study realistic scenarios by implementing several standard protocols; Open Automated Demand Response (OpenADR), Device Language Message Specification (DLMS) protocol, and an industrial aggregator $G$-smart (as seen in Figure 10); it shows the Demand/Response head-end system responsible to receive flexibility information from the customer energy management system (CEMS) that in combination with information from external factors (e.g. weather information, current market prices and more) is used by the Demand Response platform to plan production and consumption for a longer time period. Set points are then calculated to be sent to the CEMS, which then enforces the values to the household [22].

The main components and protocols of the demand management system involved as shown in Figure 10 and their functionalities are described as follows:

- Demand Management Control: calculates demand energy reference for the available assets that can control the energy load, for example CEMS or Charging stations.

- Head-end: integrates systems from the back-end with the equipments on medium voltage and low voltage levels. 


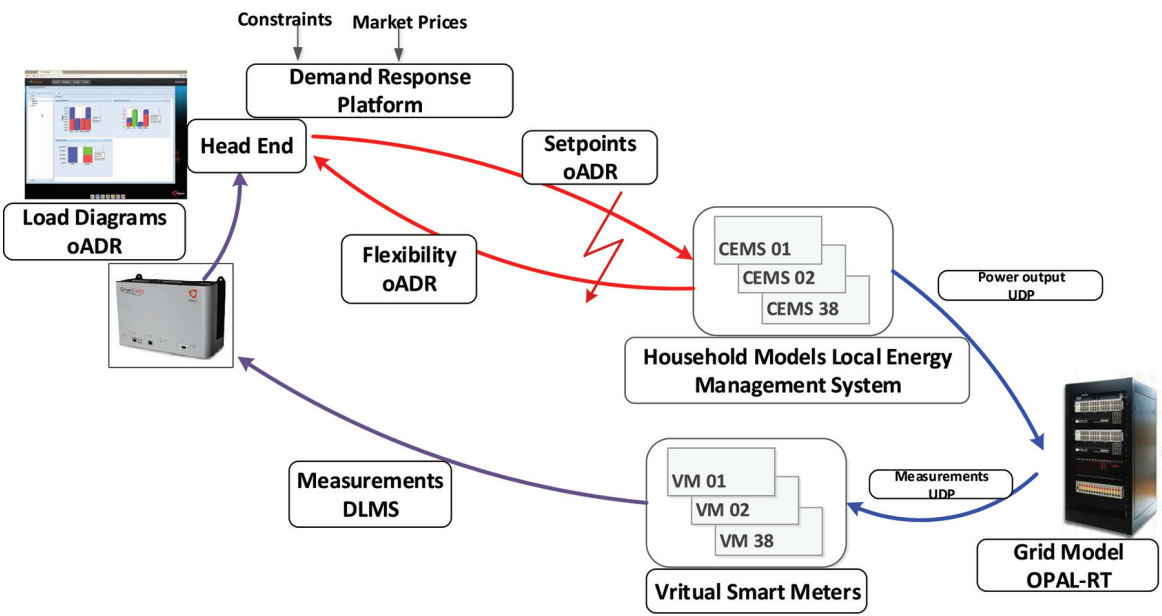

Figure 10 Demand management architecture.

- Customer Energy Management System: (CEMS), works as aggregator and controller for assets on a single household.

- Virtual Smart Meters: Communicates with OPAL-RT grid model using UDP sockets (see Section 2.3).

- G-Smart: an industrial data aggregator from EFACEC used to accumulate load diagrams from virtual smart meters implemented at virtual smart meter server and by using the standard DLMS protocol.

- Open Automated Demand Response (OpenADR): is a standard protocol used to exchange information between CEMS and the Head-end system.

- Device Language Message Specification (DLMS): is communication standard modelling the communication entities between G-Smart and virtual smart meters.

All CEMS controllers and their local asset models run on one CEMS server (see Section 2.3.3). The central DMC and the Head-end run on a dedicated machine on DSO control layer at Smart Energy Systems Laboratory (see Figure 11). The OpenADR protocol requires the definition of two additional client server pairs: the Virtual Tap Node (VTN) client \& server and the virtual end node (VEN) client \& server. In general clients have responsibility for sending messages, while the servers are responsible for listening and receiving messages (see Figure 11). At first the VTN sends a forecast of history data (background load, PV irradiation forecast and market 


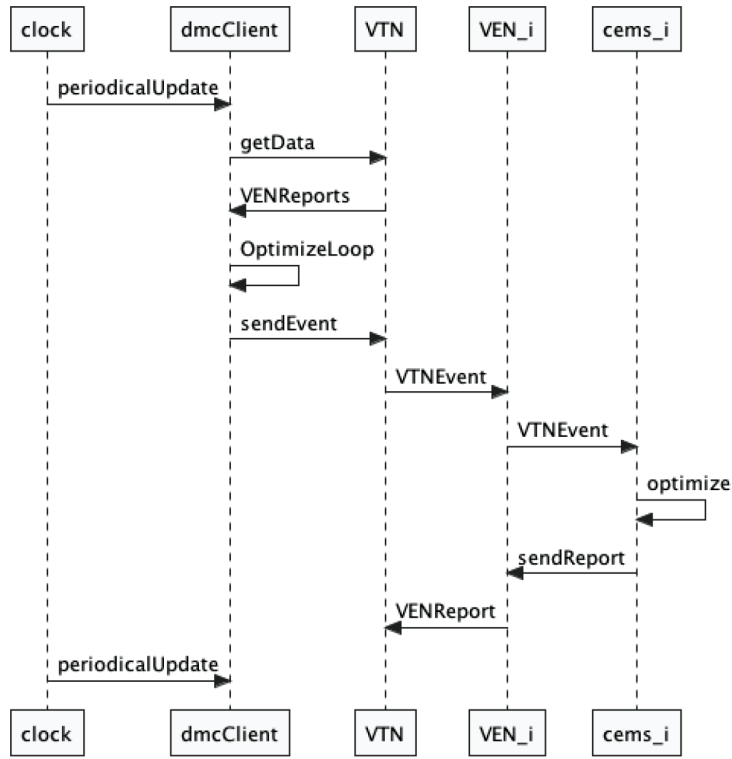

Figure 11 Interactions between the main DR components for CEMS [22].

clearing energy prices for that day). Periodical updates are initiated centrally by DMC once an hour. DMC collects the information at VTN database which also gathers the current updated information from CEMS served by VENReport. Once the data is collected the optimization loop is run over multiple iterations. Onwards, the computed setpoints are forwarded to VTN which then triggers VTNEvent for each VEN (household). The VENs call every 15 minutes to create reports and update the actuation values on CEMS.

The real-time HIL platform is used for validation of hybrid simulationbased evaluation implemented at FTW research center (a partner at SmartC2Net project). To fit with the hybrid simulation, a downscaled version of benchmark residential LV grid (see Figure 3 and Figure 4) is implemented where 38 households are connected to the low voltage grid. Real smart meter measurements are used to model the non-flexible load for each household. Each household is assumed to consume $5 \mathrm{~kW}$ for heating, ventilation and air conditioning (HVACs). PV panels have a power rating of $4 \mathrm{~kW}$. Eight houses have electric vehicle charging points associated with various parking periods. The HVAC's initial indoor temperature is randomly distributed between 19.1 and $20.9^{\circ} \mathrm{C}$, the outside temperature is assumed to be $1^{\circ} \mathrm{C}$ (January).

The experiments are performed in real-time, as CEMS setpoint consumption signals are sent to the real-time grid simulator which uses UDP sockets to 
get the values on the grid model at OPAL-RT. The virtual smart meters recreate metering information to be collected at the industrial aggregator G-Smart by using DLMS protocol and close the loop. Each test run is implemented for a span of $24+1$ hours to get valid load profiles from smart meters for the full window a complete day.

\subsubsection{Demand management test result}

To study energy cost saving by using the demand management platform; three scenarios are tested [22], with these specific test scenarios, the goal is to use local stored or produced energy internally when the price of electricity is expensive and to use more power from the grid when electricity price is low. The first scenario is tested as a measure of comparison (Baseline, fixed price and DMC in-active) for the remaining scenarios. Then the test scenario is repeated by using day ahead prices and DMC on, here an interesting correlation with the price value is observed. To study further extreme case, a scenario with day ahead prices and excessive demand on house holds is tested. Table 1 shows the overall comparison of the three cases in-terms of Energy cost saving.

Baseline with No DMC control: CEMS is not getting set-points from the DMC instead assuming constant electricity price; the consumption and generation is independent of electricity prices for the whole time span. It is used as a baseline to compare and contrast improvements in energy cost saving when we turn on the DMC.

DMC with day-ahead prices considered: DMC is sending setpoints to CEMS with day ahead electricity prices considered by the demand response platform. Figure 12 shows the results for the scenario DMC with day ahead prices (DAP) considered for CEMS on all 38 households. It shows the total load vs total setpoint of 38 houses (day ahead price considered), the load values are in 15 minute interval of the full 24 hours, 0 representing the time 00:00 [22]. Figure 13 also shows a measurement for one household. The price value is the relative increment or decrement from an average price of $44,236 \in / \mathrm{Mwh}$. As seen in the figure, the demand response platform has mitigated impact of communication overhead created by using OpenADR, DLMS protocols and

Table 1 Energy cost saving of demand response platform [22]

\begin{tabular}{llll}
\hline Scenario & Baseline & DAP & DAP Excessive Demand \\
\hline Total Energy/day & $765 \mathrm{KWh}$ & $1011 \mathrm{KWh}$ & $1118 \mathrm{KWh}$ \\
Total Cost & 33,75 & 39,70 & 48,25 \\
Resulting Avg. Price & $44,23 / \mathrm{MWh}$ & $39,28 / \mathrm{MWh}$ & $43,15 / \mathrm{MWh}$ \\
Savings & 0 & $11 \%$ & $2,5 \%$ \\
\hline
\end{tabular}




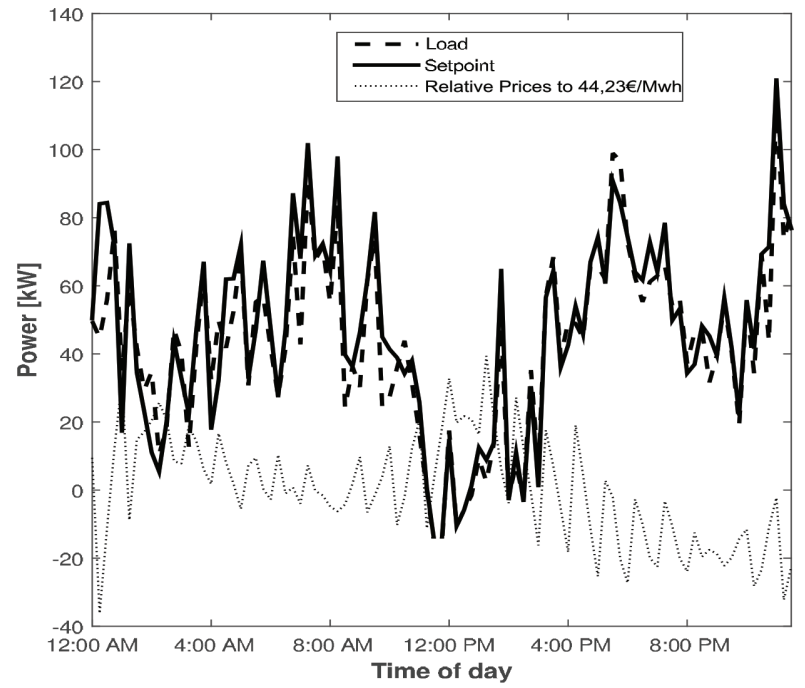

Figure 12 DMC with day-ahead price CEMS result.

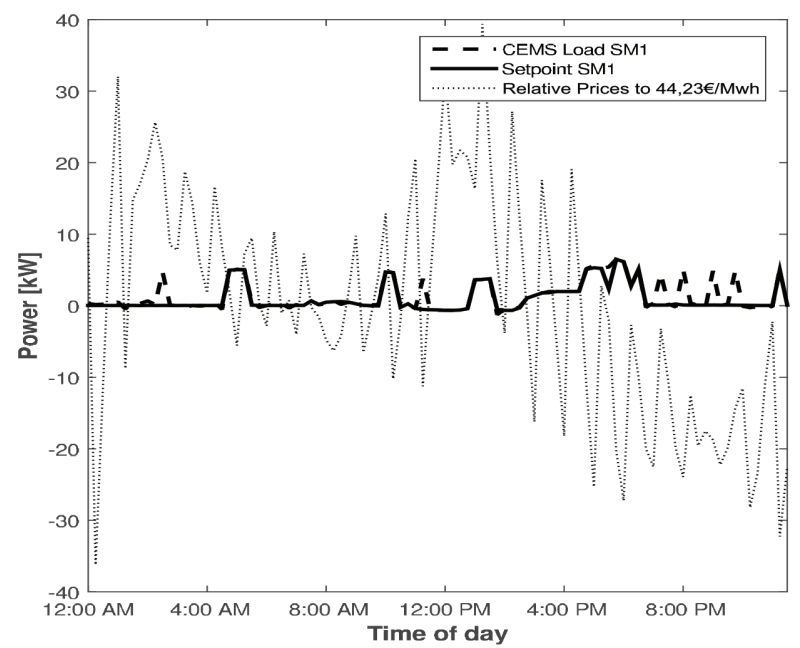

Figure 13 Household CEMS measurements.

UDP communication with the OPAL-RT grid model; i.e on reference following capacity of CEMS. We can also see an significant correlation between the price value and the set points, where in case of low price the set points increase a bit inorder to make use of the cheap electricity price from the DSO. 
The energy cost saving capacity of the system has improved by $11 \%$ as seen in Table 1 when compared to the baseline scenario.

DMC with day ahead prices and excessive demand: Overload is introduced in to the system, which means, the DMC has to decrease demand limit (LVmax ) to $70 \mathrm{~kW}$ at the transformer. This has decreased the savings down to $2,5 \%$ but still households can benefit when compared to the baseline. The full set of results is documented at [22] and will be analyzed in great detail on subsequent publication.

Impact of Demand Managment on Voltage Values: The presence of detailed power grid model on OPAL-RT has given us flexibility to study the impact of Demand Managment system on voltage traces. Two scenarios are tested, Baseline scenario with no connection to DMC and Excessive Demand with an Active-DMC set with a total power limit of PLVmax of $70 \mathrm{~kW}$. The scenarios are compared in Figures 14 and 15 showing voltage traces of Bus 14 located at the secondary side of transformer and Bus 38 connected at Feeder end. Since the real-time simulations were executed on continuous dates with different start times; Out of 24+1 hour of real-time measurements, the plots show voltage traces of 20-hour measurements with perfect time alignments for both scenarios. It can be seen from the plots that due to the impact of PV generation in the LV grid, voltages often exceed the nominal voltage value. We can also

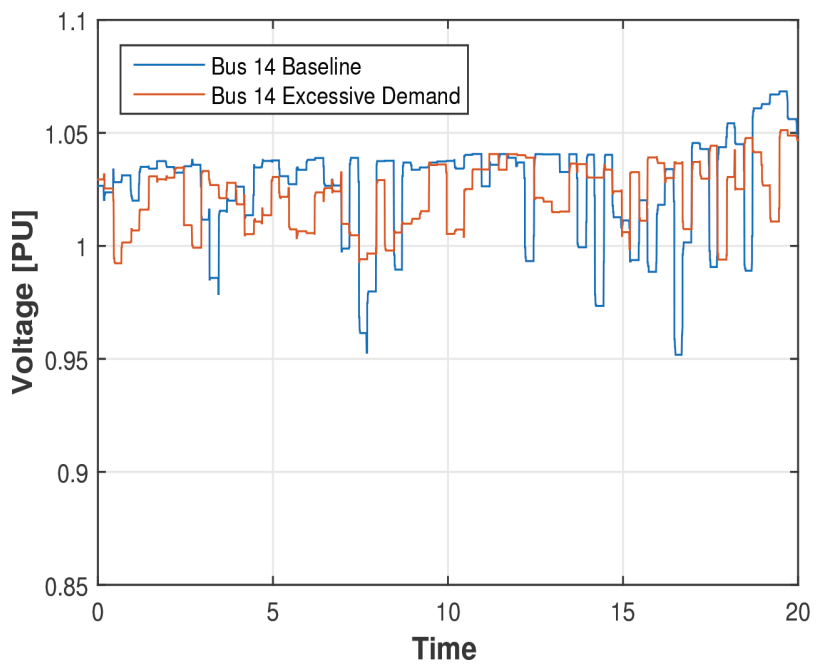

Figure 14 Bus-14 voltage traces Baseline vs. Excessive Demand at secondary side of transformer. 


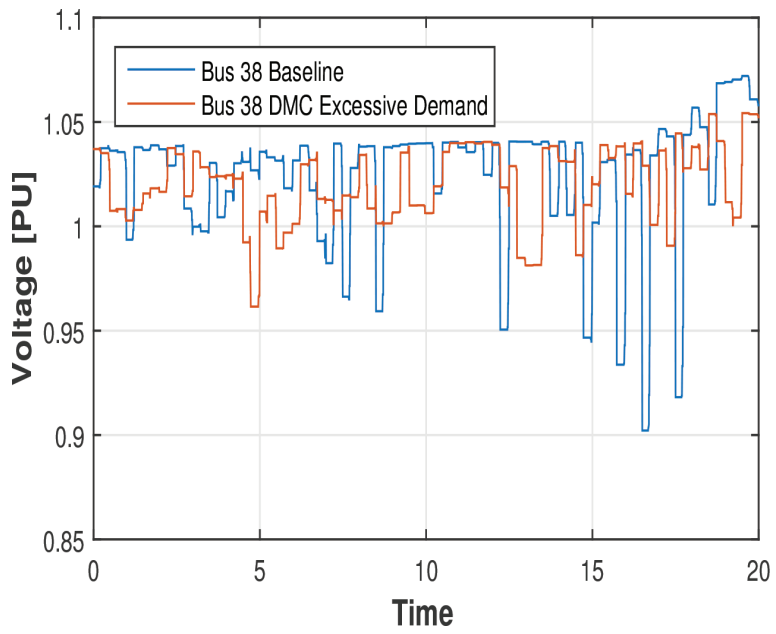

Figure 15 Bus-38 Voltage traces Baseline vs. Excessive Demand at feeder end.

see voltage drops down to $0.95 \mathrm{pu}$ of nominal fro Bus 14 and even as low as to $0.9 \mathrm{pu}$ for bus 38 which is reasonable because it is located at feeder end.

To see the voltage improvement with DMC, Figures 16 and 17 show the histogram plot of the voltage deviation index as normalized per unit voltage

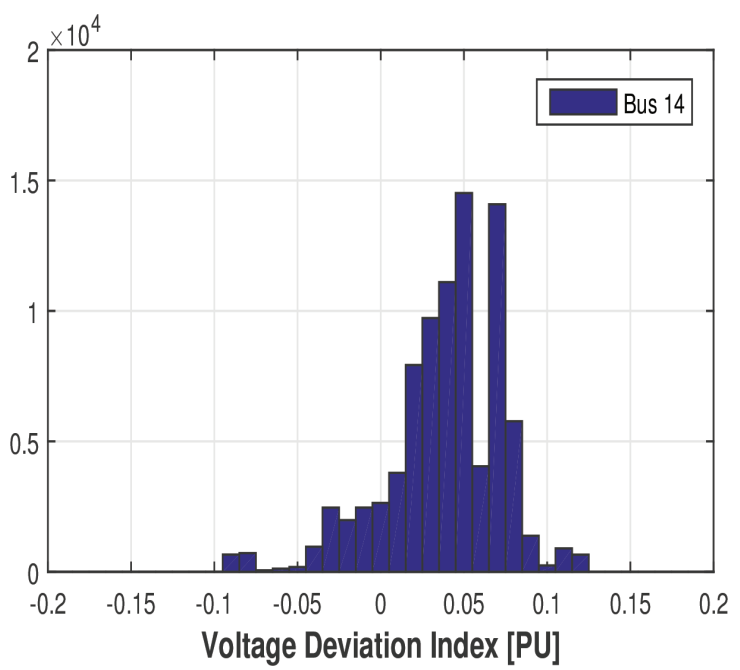

Figure 16 Bus-14 Histogram of voltage deviation index at secondary side of transformer in Excessive Demand scenario. 


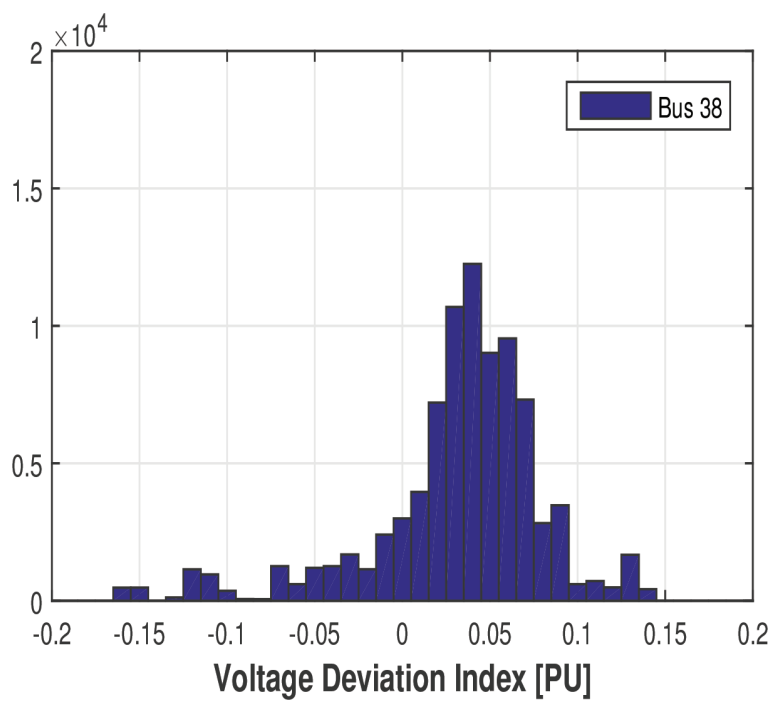

Figure 17 Bus-38 Histogram of voltage deviation index at secondary side of transformer in Excessive Demand scenario.

variation (see Equation (1) where $\mathrm{V}_{\text {base }}$ is the voltage for baseline scenario and $\mathrm{V}_{E D}$ is the voltage for excessive demand ). A positive voltage deviation shows that the DMC is improving voltage profiles compared to the baseline scenario. The plots further show even for Excessive Demand scenario, the demand management system resulted in a better performance compared to the baseline scenario. Voltage deviation for Bus 14 at the secondary side of transformer goes as low as -0.1 where for the feeder end at bus 38 it goes even lower $(-0.15)$ as it is located at feeder end.

$$
V_{d i}=\frac{V_{b a s e}-V_{E D}}{V_{b a s e}}
$$

\section{Conclusion}

This article provides a general overview of development of real-time open access platform towards proof of concept for smart grid applications at Smart Energy System Laboratory Laboratory of Aalborg University. The main components of the platform, namely physical domain comprising internal electrical grid and assets, the ICT layer, as well as the control layer are presented, and their interaction is highlighted. An innovative mechanism for setting 
and integrating of ICT and Network Emulation platform to real-time HIL smart grid laboratories is introduced. It is shown through the test case for Active Power Management in Distribution Grid how to investigate the impact of communication technologies on MV and LV controllers. The effect of communication Quality of service (QOS) parameters like delay, packet-loss, and bandwidth requirements can be studied in great detail by using the setup.

To show case how Smart Energy System Laboratory setup can be used to analyze current smart grid challenges; Active power management and Demand Management Platform implemented for European Smartc2Net project are presented. The article has shown practical implementation mechanisms taken to fulfill the vision of Smart Energy System Laboratory to capture key smart grid components and domains from energy markets down to individual smart assets in a real-time HIL framework.

\section{References}

[1] Larsen, H., Erik Morthorst, P., Bindslev, H., and Snderberg Petersen, L. (2010) "The intelligent energy system for tomorrow." 'In 21st World Energy Congress.

[2] Smart Energy System Laboratory. Available at: http://www.et.aau.dk/ department/laboratory-facilities/smart-energy-systems-lab/

[3] Dufour, C., and Blanger, J. (2014). "On the use of Real-Time Simulation Technology in Smart Grid Research and Development". IEEE Trans. Ind. Appl. 50(6), 3936-3970.

[4] Energinet Facts. Available at: http://energinet.dk/EN/El/Nyheder/ Sider/Dansk-vindstroem-slaar-igen-rekord-42-procent.aspx

[5] Pederson, R., Sloth, C., Andersen, G. B., and Wisniewski, R. (2015). "DiSC: A simulation framework for distribution system voltage control." European Control Conference (ECC). Linz, Austria.

[6] Altin, M., Hansen, A. D., Cutululis, N. A., Bindner, H. W., Iov, F., and Olsen, R. (2015). "Ancillary services from renewable power plantsRePlan project perspective." In 14th International Workshop on LargeScale Integration of Wind Power into Power Systems as well as on Transmission Networks for Offshore Wind Power Plants. 4, pp. 20-22.

[7] Chen, P., Salcedo, R., Zhu, Q., de Leon, F., Czarkowski, D., and Jiang, Z. et al. (2012). "Analysis of voltage profile problems due to the penetration of distributed generation in low-voltage secondary distribution networks". IEEE Trans. Power Delivery, 4, pp. 2020-2028. 
[8] Stergaard, J., Wu, Q., and Garcia-Valle, R. (2012). "Real Time Intelligent Control Laboratory (RT-ICL) of PowerLabDK for Smart Grid Technology Development." IEEE Com. Eng. (COMPENG), Aachen, Germany, 11-13.

[9] Deliverable 6.3 SmartC2Net, (2015) "Smart Control of Energy Distribution Grids over Heterogeneous Communication Networks." Aalborg, Denmark.

[10] DiSC-A Simulation Framework for Distribution System Voltage Control, (2014). Available at: http://kom.aau.dk/project/SmartGridControl/ DiSC/

[11] Kemal, M. S., Pedersen, R., Iov F., and Olsen R. (2017). "DiSC-OPAL: A Simulation Framework For Real-time Assessment of Distribution Grids." Workshop on Modeling and Simulation of Cyber-Physical Energy Systems.

[12] Bianchi, F. D., de Battista, H., and Mantz Wind R. J. (2017). "Turbine Control Systems: Principles, Modelling and Gain Scheduling Design." Advances in Industrial Control. Springer.

[13] der Hoven I. V., (1957). "Power spectrum of horizontal wind speed spectrum in the frequency range from 0.0007 to 900 cycles per hour." $J$. Meteorol. 14, pp. 160-164.

[14] Villalva, M. G., Gazoli, J. R., and Filho E. R. (2009). "Comprehensive approach to modeling and simulation of photovoltaic arrays." IEEE Trans. Power Electr. 24, 1198-1208.

[15] Deshmukh M. K., and Deshmukh, S. S. (2008). "Modeling of Hybrid Renewable Energy Systems." Renewable and Sustainable Energy Reviews. 12, 235-249.

[16] National travel survey Department for Transport, (2012). Tech. Rep., Available at: https://www.gov.uk/government/publications/nationaltravel-survey-2012

[17] Kundur P. (1993). "Power System Stability and Control." The EPRI Power System Engineering Series. McGraw-Hill.

[18] Kemal, M., Iov, F., Olsen, R., Le Fevre, T., and Apostolopoulos, C. (2015). "Online Configuration of Network Emulator for Intelligent Energy System Testbed Applications." IEEE AFricon. Addis Ababa, Ethiopia, 1-4.

[19] Garcia, J., Hurtig, P., and Brunstrom A. (2008). "A Versatile and Flexible Emulation System 2015 Swedish National Computer Networking Workshop (SNCNW08)." Sweden. 
[20] Cisco Catalyst 2960xseries Switch. Available at: http://Cisco.com

[21] Bessler, S., Kemal, M. S., Silva, N., Olsen, R., Iov, F., and Drenjanac, D. (2017). "Grid-Aware Demand Management in Electricity Distribution Grids. Sustainable Energy." Grids and Networks.

[22] Smart Control of Energy Distribution Grids over Heterogeneous Communication Networks. (2012). Available at: http://www.smartc2net.eu/

\section{Biographies}

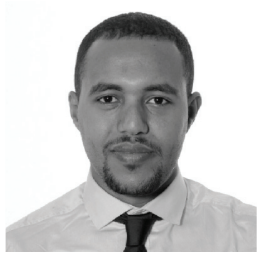

Mohammed Kemal received his B.Sc degree in Electrical, Electronics and Communications Engineering from Jimma University, Ethiopia and M.Sc degree in Network and Distributed Systems from Aalborg University, Denmark in 2008 and 2014, respectively. From 2008 to 2012, he was working as a Telecom Engineer at Cresent Engineering and IT Solutions, Ethiopia. where he was responsible for design, installation and monitoring of network and communication systems for multipurpose industrial infrastructures. In 2014-15, He has worked a Scientific Assistant at Department of Energy and Technology Aalborg University, and currently pursuing his $\mathrm{Ph} . \mathrm{D}$ in the area of adaptive data monitoring for Smart Metering infrastructures at Department of Electronics of Aalborg University, Denmark. His current research interests include Integration of ICT and Communication systems for Smart Grid Platforms, Automatic Metering Infrastructures, Demand Response Platforms and SCADA systems. 


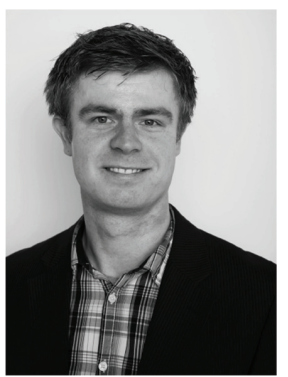

Lennart Petersen was born in Schluechtern, Germany, in 1989. He received the Ba.Eng. degree in Renewable Energy Technologies from the University of Applied Sciences in Flensburg, Germany, in 2013. In 2012, he joined the wind turbine manufacturer Senvion SE (former REpower Systems SE) in Buedelsdorf, Germany, as a trainee, where he has drawn up his Bachelor thesis and has continued to work as a student employee until 2015. Since September 2013, he has been with the Department of Energy Engineering, Aalborg University, where he has completed his Master studies in Wind Power Systems until June 2015. At the moment he is working as research assistant at Aalborg University in the field of Electrical Power Systems. His current research topics include voltage and frequency control in transmission and distribution grids with high penetration of renewable energy sources as well as harmonics analysis in wind power plants.

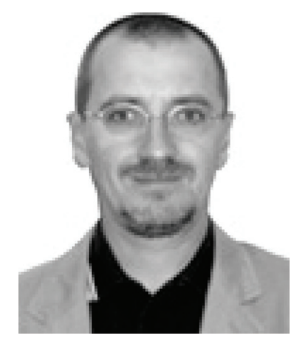

Florin Iov (S'98-M'04-SM'-06) received the M.Sc degree in Electrical Engineering from Brasov University, Romania, in 1993 and a Ph.D degree from Galati University, Romania in 2003 with a special focus in the modeling, simulation and control of large wind turbines. He was staff member at Galati University, Romania from 1993 to 2001. Dr. Iov was with Institute of Energy Technology, Aalborg University, Denmark between 2001 and 2009 where he was mainly involved in research projects regarding grid integration of wind 


\section{M. Kemal et al.}

power. From 2010 to 2012 he held a position as Power System Research Specialist in Vestas Wind Systems working with new ancillary services for augmented wind power plants. Since 2013 Dr. Iov is with Institute of Energy Technology focusing on research within smart grids. His research areas covers control and application of electrical machines and power electronic converters for grid integration of renewable energy sources and, operation and control of dispersed generation in modern power systems. He is author or co-author of more than 120 journal/conference papers.

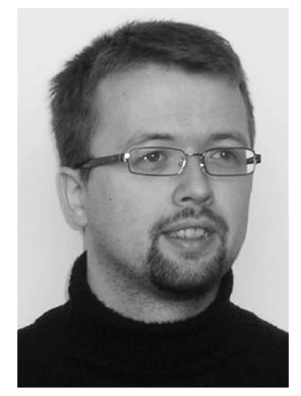

Rasmus Løvenstein Olsen is an Associate Professor at department of Electronic systems, Aalborg University. Rasmus received his master degree from Aalborg University in 2003 and has received his Ph.D degree on the topic of Context Sensitive Service Discovery and Context Management with focus on access to dynamic information in 2008. Since then, Dr. Olsen have been teaching, supervising and working in various European projects and have more than 40 publications in papers for international conferences, journals and book chapters. Rasmus current research focus is on service migration based on context information, context quality metrics and quality of service in context management systems, as well as usage of dynamic information elements in smart grid scenarios. 